\title{
Virtual rounds: Observational study of a new service connecting family members remotely to inpatient rounds
}

\author{
Kristin L. Rising, ${ }^{1,2}$, Julianna C. Ricco ${ }^{1}$, Alexandra D. Printz ${ }^{2}$, Sang Hoon Woo ${ }^{3}$ and Judd E. Hollander ${ }^{1,2}$ \\ ${ }^{1}$ Department of Emergency Medicine, Thomas Jefferson University, Philadelphia, USA \\ ${ }^{2}$ National Academic Center for Telehealth, Thomas Jefferson University, Philadelphia, USA \\ ${ }^{3}$ Division of Hospital Medicine, Thomas Jefferson University, Philadelphia, USA
}

\begin{abstract}
Background: Family members provide important support for patients, yet there has been minimal focus on including family and other caregivers in medical discussions. Objective: To report on a pilot program, virtual rounds, designed to facilitate remote inclusion of family members during daily medical rounds.

Design: Prospective descriptive study of virtual rounds implementation.

Setting: An academic tertiary care hospital in Philadelphia.

Participants: 218 patients admitted to the oncology service: age range $22-89$ years, $51 \%$ female.

Interventions: Participation in virtual rounds, a service with which family members or other individuals identified by the patients could be included in upcoming rounds with the medical care team.

Measurements: Number of patients approached who were interested in and completed a virtual round; relationships of patient-identified participants; reasons for declining participation.

Results: Out of 218 approached patients, 91 agreed to participate (42\%) and $48(22 \%)$ completed one or more virtual rounds. Half of patients (N=107, 49\%) declined participation, with reasons including 1$)$ no perceived need $(n=58,54 \%) ; 2)$ no available equipment ( $n=10,9 \%)$; and 3) no interest $(n=30,28 \%)$.

Limitations: Small sample size, single oncology hospital service, and single institution study. No health-related outcomes or patient satisfaction measures.

Conclusions: Less than half of patients were interested and less than one quarter of patients actually received the service, though close to half of patients receiving the service requested repeat utilization. Patient-centered delivery system must be flexible and filled with options, as no two patients are the same and thus preferences for care will vary.
\end{abstract}

\section{Introduction}

Delivery of more patient-centered healthcare has been a national priority for the past decade [1,2]. As patients are increasingly empowered to take a more active role in healthcare decisions, they may benefit from inclusion of their primary social support figures in discussions regarding medical care.

Common sense dictates that engaging family members, along with the patient, when discussing important medical information should result in improved knowledge retention. Family and other caregivers may also personally benefit from improved communication regarding patients' care [3]. Studies have shown that family members are not satisfied with the level of communication they receive from hospital staff. In one study, $54 \%$ of family members reported trouble comprehending diagnosis, prognosis, or treatment of their family member [4]. Family-centered rounds implemented in the intensive care unit (ICU) at Vanderbilt University Hospital resulted in higher family satisfaction [5]. Qualitative interviews with cancer patients and families emphasized the need for effective communication. Patients who participated in these studies were enthusiastic for new methods of monitoring and improving communication with family members $[6,7]$.

Despite findings from these studies, there has been minimal focus on expanding capabilities of the medical system to include family and other caregivers in discussions of patients' healthcare, especially when patients are hospitalized and loved ones are not physically present. In response to this unmet need, we implemented virtual rounds, with which family members can remotely participate during daily patient rounds with the healthcare team. We report initial experiences with implementation of virtual rounds, including patient uptake rates and barriers to implementation.

Correspondence to: Kristin L. Rising, MD, MS, Director of Acute Care Transitions \& Assistant Professor, Department of Emergency Medicine and the National Academic Center for Telehealth, Thomas Jefferson University, Philadelphia, PA, USA, Tel:215-503-5507, Fax: (215) 952-5193; E-mail: mailto:Kristin.Rising@jefferson.edu

Received: February 24, 2016; Accepted: April 12, 2016; Published: April 15, 2016 


\section{Materials and methods}

\section{Study design and setting}

This is a prospective descriptive study of virtual rounds implementation at Thomas Jefferson University Hospital, an academic tertiary care hospital in Philadelphia.

\section{Selection of participants}

Patients 18 and older who were hospitalized were eligible for virtual rounds. Virtual Rounds was initiated on the oncology service, which is a co-managed service with oncologists and hospitalists. This service was geographically located within the institution and already conducted patient-centered multi-disciplinary rounds before implementation of virtual rounds.

Patients on contact precautions were excluded due to initial concerns about sufficient iPad sanitation and limited availability of team members trained to interact with patients on contact precautions. Patients who did not speak English fluently or who were deaf were also excluded.

All patients, family members, and friends gave verbal consent for participation in virtual rounds. Because this was a clinical program that was collecting only performance improvement related information, the institutional review board determined it was exempt from formal review.

\section{Intervention}

Patients and family members: Eligible patients were approached by members of their care team or by telehealth coordinators to assess their interest in participation in virtual rounds. As explained to patients, virtual rounds offered a means of remotely connecting family or other caregivers to the daily patient medical rounds.

Implementation of virtual rounds was facilitated through telehealth coordinators, who were individuals trained in use of the videoconferencing platform. Coordinators approached eligible patients daily to provide information about virtual rounds. Interested patients were asked to identify the individuals they would like virtually connected during the next day's morning rounds and to provide relevant contact information for these individuals. Identified participants were then contacted by phone to be invited to participate in the virtual rounds service and to be educated about use of the video-conferencing software.

Individuals were guided through the process of either downloading the application to their smartphone or tablet or instructed on how to access the platform on their computer. They were given a time range for when the rounds would take place the following day, and were texted or called the following day to alert them as to a more precise time of rounds.

Technology: The service was provided with use of the Blue Jeans video-conferencing platform. Blue Jeans is a HIPPA-compliant cloudbased video service allowing for multiple party connections across several platforms. Individuals identified by the patients could establish a virtual connection with the patient's room while the medical providers were physically present during daily rounds, thus participating in the daily medical discussions.

\section{Data collected}

All patients approached were recorded in a database. Data collected included patient name, date approached, individual who approached the patient, response from patient (yes/no) and reason for response, and contact information for patient-identified participants. Visit data collected included date visit was scheduled to occur, attending on service, completion status, family device used, number of people connected, and call duration. If a scheduled virtual round was not completed, the reason for this was also recorded.

\section{Outcomes measures and analysis}

The primary outcome reported is the proportion of total number of patients approached who were interested in and completed a virtual rounds session. In addition, we report the relationships of patientidentified participants who were included in completed virtual rounds sessions as well as the most prevalent reasons cited by patients who said no to the service. Analysis is descriptive, including counts and percentages.

\section{Role of the funding source}

This program received internal funding from Thomas Jefferson University in the form of salary support for staff, licensing fees for software, and hardware support for the technology.

\section{Results}

There were a total of 298 oncology admissions during the 3-month study period. There were 80 patients who met exclusion criteria and were not approached for participation, leaving 218 patients who were approached for inclusion. Out of these, $42 \%$ (91/218) agreed to participate, and over half of these patients $(53 \%, 48 / 91)$ completed one or more virtual rounds. Forty percent (19/48) of patients who participated completed more than one call. Fifteen attending physicians participated in virtual rounds during the study period (Figure 1).

Patients who completed virtual rounds spanned 6 decades of life and most often included their children in the discussion (Table 1).

About half of patients $(107 / 218,49 \%)$ declined participation in the service. The general categories of reasons provided for lack of interest in the service were 1) no perceived need; 2 ) no available equipment; and 3) no interest. The most common reason provided was no perceived need for the service because family or other caregivers were already adequately engaged in their healthcare (Table 2).

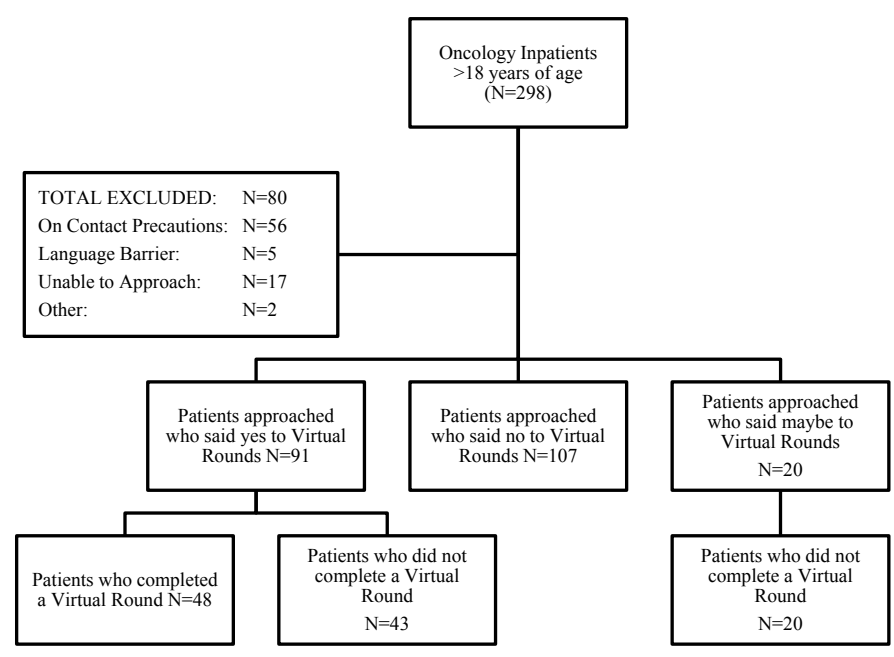

Figure 1. Patient flow chart. 
Table 1. Patient demographics.

\begin{tabular}{|c|c|c|}
\hline Demographics & Patients Approached $(\mathrm{n}=\mathbf{2 1 8})$ & $\begin{array}{l}\text { Patients Completed } \\
\text { Virtual Rounds }(n=48)\end{array}$ \\
\hline Age, mean (years) & 62.9 & 59.2 \\
\hline Age, range (years) & $22-89$ & $26-87$ \\
\hline Female & $112(51 \%)$ & $28(58 \%)$ \\
\hline Length of Stay (days) & 9 & 12 \\
\hline $\begin{array}{l}\text { Patient-identified participants }(\mathbf{n}=\mathbf{5 8}) \\
\text { - Spouse } \\
\text { - Daughter } \\
\text { - Son } \\
\text { - Sibling } \\
\text { - Parent } \\
\text { - Friend } \\
\text { - Other/unknown }\end{array}$ & & $\begin{array}{l}9(16 \%) \\
22(38 \%) \\
7(12 \%) \\
7(12 \%) \\
5(9 \%) \\
5(9 \%) \\
3(5 \%)\end{array}$ \\
\hline
\end{tabular}

Table 2. Reasons provided by patients for declining use of virtual rounds ( $\mathrm{n}=107)$.

\begin{tabular}{|c|c|}
\hline Reasons Provided & n (\%) \\
\hline 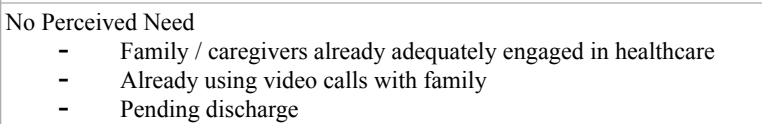 & $\begin{array}{l}39(36 \%) \\
3(3 \%) \\
16(15 \%)\end{array}$ \\
\hline $\begin{array}{l}\text { No Available Equipment } \\
-\quad \text { No family access to device, general discomfort with tech }\end{array}$ & $10(9 \%)$ \\
\hline $\begin{array}{cl}\text { No Interest } & \\
- & \text { General disinterest } \\
- & \text { Didn't want family involved / no family members to contact } \\
- & \text { Discomfort with video due to symptoms of illness (i.e. vomiting) }\end{array}$ & $\begin{aligned} & 18(17 \%) \\
& 8(7 \%) \\
& 4(4 \%)\end{aligned}$ \\
\hline No Reason Provided & $9(8 \%)$ \\
\hline
\end{tabular}

Challenges encountered that prevented successful completion of virtual rounds for patients who wanted them included: inability to contact the individuals identified by patients for inclusion; lack of access to proper technology by the individuals contacted and time conflicts between the individuals contacted and the medical teams.

\section{Discussion}

We report here the results of implementation of a new patient- and family-centered service, virtual rounds, which was designed to improve communication between patients, caregivers, and providers. We anticipated that the majority of patients would want this service, yet less than half of patients approached were interested in the service. In addition, despite extensive implementation planning prior to initiation of the service, less than half of those patients interested in the service actually completed at least one virtual round.

We suggest a number of important learnings from this pilot program. First, we address the discrepancy between expected and actual patient interest. While close to half of patients were interested, and most who had one visit requested repeat visits, the other half were not interested for various reasons. Many patients declined the service because their family or caregivers were able to be present in person. Others had technical barriers, lack of desire for family involvement, or just simple lack of interest. These findings highlight the fact that all patients are not the same, and that there is not a one-size-fits-all approach to healthcare delivery. Thus, our future efforts are focused on how to streamline the process of identifying those patients who are interested in the service instead of trying to modify the service to appeal to all patients. We now have intake nurses assessing initial patient interest for virtual rounds during hospital admission, thus focusing telehealth coordinator efforts on those patients most interested in the service.
Second, it is important to determine the barriers to implementation for those patients who were interested in the service but were unable to receive it. Offering a service that we were then unable to provide was clearly not patient-centered, so we worked to address this problem as efficiently as possible. We found that despite family members confirming participation the night prior to rounds, they often did not participate. Thus, we started to send text messages to loved ones the morning of the rounds to confirm participation. For those family members who needed it, we also provided video test calls the night prior to the scheduled rounds. Though we were unable to address lack of technology access on the end of the remote family members, we were able to provide extended support for those who had the technology though felt unsure about how to use it to access this service.

We continue to offer and expand this service, and are constantly refining our approach to scaling up into integrated, hospital-wide program. Intake nurses now identify all interested patients at the point of admission, with responses feeding into a central dashboard. Telehealth coordinators then approach interested patients to provide further education and to gather the contact information of individuals to be included in the rounds. This approach, along with increased telehealth coordinator staffing to cover 12-16 hours per day on weekdays, has significantly increased our reach throughout the hospital. Our eventual goal is that intake nurses will gather contact information themselves and will send information to family members for self-education about how to download and connect to virtual rounds. This would eliminate the need for telephone calls between participants and team members prior to the actual virtual rounds sessions and reduce the staffing needs.

We suggest a few important considerations for those who are considering implementation of a similar service in their facility. Support for this initiative at all levels, from administration through faculty and other staff, is vital for successful implementation. Clinician 
champions are crucial in educating providers and patients about the benefits of participation and in addressing concerns of potential late- or non-adopters. Developing a network of champions prior to implementation is something any future pilot service should consider. Early involvement of legal services is also important in establishing telehealth consent procedure in compliance with the hospital's legal counsel before any patients are involved. Perhaps most importantly, the human resources needed to make initial implementation successful are significant. We had six telehealth program personnel participating in these tasks on top of their other responsibilities over the course of the study period, with 2-3 people managing each week's workload. While we do believe that this program can be integrated into standard hospital procedures over time, the initial implementation period does require significant hands-on time investment from a dedicated core of individuals.

\section{Limitations}

Our study is limited by both a relatively small sample size as well as the inclusion of patients on a single oncology hospital service. Our goal with this report, however, is primarily to report the findings of the first few months after piloting this service. We do not suggest that our findings are representative of all populations, and acknowledge that responses from patients with a variety of conditions over a broad age range would likely elicit different results. For example, we note that some patients within this study population reported their specific disease symptoms, including persistent vomiting and looking generally unwell, as deterrents to participating in this service. Further research into the acceptability and feasibility of implementing this service among different patient populations and hospital services is needed.

Finally, the study does not report on any health-related outcomes or patient-satisfaction associated with utilization of virtual rounds. These measures will eventually warrant further study as virtual rounds expands.

\section{Conclusion}

We implemented a new service designed to improve our delivery of patient-centered care, and found that less than half of patients were interested in the service and less than one quarter of patients actually received the service. We suggest that these findings support the idea that a patient-centered delivery system must be flexible and filled with options, as no two patients are the same and thus preferences for care will vary. We continue to move forward in efforts to expand through all hospital wards and integrate the process into standard hospital workflows, while also engaging patients to determine other ways in which we can build a more flexible and responsive patient-centered delivery system.

\section{Authorship \& contributions}

$\mathrm{KR}, \mathrm{SW}$ and JH conceived of and designed the intervention. JR and AP performed intervention implementation and data acquisition. All authors contributed to manuscript drafting and revision, and provide final approval of the submitted version. KR takes responsibility for the manuscript as a whole, though all authors have agreed to act as guarantors of the work.

\section{Acknowledgements}

None

\section{Funding}

None

\section{Competing interests}

The auhors declare that they have no competing interests.

\section{References}

1. Institute of Medicine(2001)Crossing the quality chasm: a new health system for the 21 th century. Washington (DC): National Academies Press (US). [Crossref]

2. Hickam D, Totten A, Berg A, Rader K, Goodman S, Newhouse R (2013) The PCORI Methodology Report PCORI Methodology Committee.

3. Auerbach SM, Kiesler DJ, Wartella J, Rausch S, Ward KR, Ivatury R (2005) Optimism, Satisfaction With Needs Met, Interpersonal Perceptions of the Healthcare Team, and Emotional Distress in Patients' Family Members During Critical Care Hospitalization. Am J Crit Care 14:202-210. [Crossref]

4. Azoulay E, Chevret S, Leleu G, Pochard F, Barboteu M, et al. (2000) Half the families of intensive care unit patients experience inadequate communication with physicians. Crit Care Med 28:3044-3049. [Crossref]

5. Jacobowski NL, Girard TD, Mulder JA, Ely W (2010) Communication in Critical Care Family Rounds in the Intensive Care Unit. Am J Crit Care 19:421-430. [Crossref]

6. Mazor KM, Gaglio B, Nekhlyudov L, Alexander GL, Stark A, et al. (2013) Assessing patient-centered communication in cancer care: stakeholder perspectives. $J$ Oncol Pract $9: \mathrm{e} 186-\mathrm{e} 193$. [Crossref]

7. Mazor KM, Beard RL, Alexander GL, et al. Patients' and Family Members' Views on Patient-Centered Communication During Cancer Care. Psychooncology. 2013;22(11). [Crossref]

Copyright: (C2016 Rising KL. This is an open-access article distributed under the terms of the Creative Commons Attribution License, which permits unrestricted use, distribution, and reproduction in any medium, provided the original author and source are credited. 